\title{
VIEWPOINT
}

\section{A handoff is not a telegram: an understanding of the patient is co-constructed}

\author{
Michael D Cohen*1, Brian Hilligoss² and André Carlos Kajdacsy-Balla Amaral*3
}

\begin{abstract}
Hospital handoffs are believed to be a key locus of communication breakdown that can endanger patient safety and undermine quality of care. Substantial new efforts to better understand handoffs and to improve handoff practices are under way. Many such efforts appear to be seriously hampered, however, by an underlying presumption that the essential function of a handoff is one-way information transmission. Here, we examine social science literature that supports a richer framing of handoff conversations, one that characterizes them as co-constructions of an understanding of the patient.
\end{abstract}

\section{The importance of handoff communication}

Over the course of their stay, in-hospital patients are seen by multiple providers. As patient care responsibility is transferred or shared among different services in the hospital and among different health-care professionals during successive work shifts, the communication of information pertinent to patient care is fundamental for continuity of care. This process is known as 'handoff' and by closely related names, such as 'sign-out,' 'sign-over', or 'handover.' We define a handoff as the information exchange that takes place when a new clinician assumes control of, or takes responsibility for, a patient. The primary focus of a handoff is to provide patient information that will allow increased effectiveness and safety of actions by the receiving party [1].

Handoffs entail a very complex process, not regularly taught during medical education [2], making them a weak link in quality and patient safety [3-5]. An analysis

*Correspondence: mdc@umich.edu; andrecarlos.amaral@sunnybrook.ca 'School of Information, University of Michigan, 3442 North Quad, 105 South State Street, Ann Arbor, Ml 48109-1285, USA

${ }^{3}$ Department of Critical Care Medicine, Sunnybrook Health Sciences Centre, University of Toronto, 2075 Bayview Avenue, Room D108, Toronto, ON M4N 3M5, Canada

Full list of author information is available at the end of the article of the quality of the care provided by cross-covering teams and an analysis of episodes resulting in malpractice suits suggest an association between handoffs and unwanted outcomes. A twofold increase in preventable adverse events has been demonstrated in patients being covered by on-call physicians belonging to a team different from the daytime care team [6]. Handoffs were implicated in $28 \%$ of surgical errors [7] and in $20 \%$ to $24 \%$ of malpractice claims in health-care settings such as ambulatory care [8] and emergency [9] departments.

This concern with accurate and complete information transfer has been a central focus of handoff research and of policy recommendations, such as the Patient Safety Requirement, which was promulgated by The Joint Commission (Oakbrook Terrace, IL, USA) and which requires US hospitals to standardize the way handoffs are performed [10]. However, it is becoming increasingly clear that this underlying presumption of one-way information transmission is seriously hampering efforts to understand and improve handoffs [11-13].

Of course, accurate transmission of key facts is very important. It is clearly problematic if the oncoming caregiver receives an erroneous report of a drug dosage or is not told that the patient should not be resuscitated. Many proposed handoff systems recognize such dangers and include remedies such as 'read-back' [14-16] or providing a clear opportunity for questions [10,17]. We certainly would not argue that accurate transmission is undesirable. We argue only that accurate transmission does not suffice to guarantee that handoffs will accomplish their larger purpose of creating in the mind of the oncoming party a fuller comprehension of the most important and uncertain aspects of the patient's course. When practitioners focus unduly on the transmission of information, handoffs are, many times, like telegrams. For example, in the majority of clinical handoffs from internal medicine residents, no questions were asked by the incoming party [18]. One may argue that the lack of questions may be due to previous knowledge of the patient; however, in the same study, the authors demonstrated that important clinical information was omitted or corrupted in more than $20 \%$ of sequential handoffs [1]. Similarly, an undue emphasis on one-way transmission 
can lead to research designs and policy recommendations that fail to recognize the active participation of both parties in co-constructing the oncoming caregiver's understanding of the patient. The remainder of our viewpoint article presents this idea and some of the findings from research outside medicine that support and refine it.

\section{Effective communication is far more than one-way transmission}

When we talk about handoffs with physicians, nurses, and technicians and when we watch clinicians at work, we do see that they are concerned to hear about important tests and treatments and to transmit to-do items and the background data that support those plans. But they also display a keen interest in getting what is often called 'the big picture.' Imagine the example of a 63-year-old man with septic shock. Different types of information will be exchanged during the handoff of this patient:

1. Background clinical information ('He is known to have COPD [chronic obstructive pulmonary disease] and hypertension.)

2. Course of the acute illness ('This is a patient with septic shock from perforated ischemic bowel, who had acute lung injury and acute kidney injury. He has been extubated for the past 24 hours and is starting to diurese spontaneously.)

3. To-dos (clear tasks that need to be completed in a certain time frame, such as 'He needs to have a new catheter inserted to re-start dialysis tomorrow')

4. Uncertainty ('He was slightly hypotensive overnight. I think we might have made him hypovolemic with the ultrafiltration. He was on a low dose of pressors this morning, but he is off pressors now after a fluid challenge. I am not sure whether he is becoming septic again')

5. Anticipation of events ('In case his blood pressure drops again, I'd re-start antibiotics and arrange for an abdominal CT [computed tomography] scan'.)

These common features of the handoff conversation show that it orients the oncoming party toward the patient, sensitizes the new caregiver to some events that may be important signals, and implicitly steers other issues toward the background. The crucial feature of this aspect of handoff is that it shapes the viewpoint of the receiving party, situating the patient within existing health-care practices and knowledge and altering the framework of expectations within which new events will be perceived. This is actually the root meaning of to inform, 'to give shape to' an actor's subsequent interpretations. It is a far deeper sense of information than the more common image that we often fall back on, that of transmitted data, as when text characters or other signals are moved along a wire or an optical fiber, as conceived in the engineering approach to communication which stems from the seminal work of Claude Shannon [19]. What ultimately matters is not just the completeness, or even the detailed accuracy, of what arrives at the other end, instrumental though these data items may be. Indeed, as electronic records of patients make the basic data ever more widely accessible, these data become less crucial in a handoff. Rather, what matters most is the effect of handoff interaction on the mind of the receiver, on the subsequent ability to make sense of the patient's unfolding episode of illness and treatment and to take the appropriate actions. In fact, we have evidence that handoffs are ineffective in having this shaping effect on the receiver's mind: physicians agreed on the main problem of patients admitted from an emergency department to hospital wards in less than $50 \%$ of handoffs, doing worse with patients who were more complex [20]. This has obvious implications for critical care patients, as they are all complex. To see how the same information can influence the receiver's perception of clinical situations in different ways, suppose that the physician in the previous example said simply, 'Oh, and he was just a bit dry overnight', which identifies the same issue (hypovolemia) but with a different emphasis on the uncertainty and its diagnostic and treatment implications.

Once we acknowledge this crucial function of handing off, it is clear that we need to focus not only on what is transmitted by the so-called 'sender' in the handoff but just as heavily on actions of the newly responsible party and on the structure of the interaction between them. Many substantial lines of social science research show that the effectiveness of communication in altering subsequent action depends on the knowledge state [21] and the active engagement of the receiving party and on the unfolding interaction pattern among those taking part in the communication, an interaction that may be affected by differences in the prior experience, social status, motivation, or specialized training of the participants [22-27].

\section{What can be learned from cognitive research for handoffs?}

To understand how one can promote deeper understanding, we must first consider how knowledge is stored in memory and how it changes when faced with new information. Decades of cognitive research have explored the concept of mental models. Seminal work by JohnsonLaird in the early 1970s introduced this concept: 'It is possible that from the meanings of sentences in a connected discourse, the listener implicitly sets up a much abbreviated and not especially linguistic model of the narrative, and that recall is very much an active reconstruction based on what remains of this model' [28].

This theory becomes crucial during handoffs, for two reasons. First, given that critically ill patients are 
inherently complex, it is likely that a single person may not generate some important possible interpretations. Having a second participant with different mental models can help generate scenarios that were not initially considered. Second, the person handing off the patient possesses a large amount of information summarized according to his or her own mental model; the ability to communicate this information to the next person is facilitated by sharing similar mental models or can be hindered if the mental models are very different.

In any given handoff, two possibilities exist: the mental models of participants are either different or similar. From the above argumentation, it can be seen that different mental models are not necessarily good or bad but that the way they are handled during a handoff is what actually matters. When different mental models are present, they may hinder efficiency (by requiring more time for explanation) or may be unsafe as the receiving party may not fully understand the patient if not enough explanation is given. However, when there is enough uncertainty about a patient (as frequently occurs in critically ill patients), more discussion and argumentation can, indeed, generate new possibilities for patients, leading to the co-construction of clinical understanding. In fact, especially when a diagnosis is unclear, a discussion between two participants with similar mental models may be less safe, as both may be agreeing on an incorrect diagnosis. This concept, of confronting different mental models to generate a novel perspective, has rarely been studied in the handoff literature.

On the other hand, there is a large body of work that studies the situation in which mental models are similar: the concept of a team mental model, also known as a 'shared mental model' [29]. When participants of teams have similar mental models, these teams can perform more efficiently and communicate more consistently with others such as consultants or family members. Indeed, a meta-analysis of published work on the team mental model and team performance across diverse areas, such as military, sports, and aviation, shows that up to $30 \%$ of a team's performance can be explained by having similar mental models [30]. Some health-care areas, such as trauma simulation [31] and laparoscopic surgery [32], have already described associations between team mental model and performance. Although participants of a handoff will not act as formal teams (as they do not provide care at the same time), they can be seen as a broader team, one that provides continuity of care over time.

\section{From individual mental models to establishing common ground}

Our views of the importance of mental models, therefore, go beyond simply stating that similar mental models may be 'better' than different mental models. As there are pros and cons of having similar or different mental models, we argue that the fundamental aspect of a handoff is in using the mental models of all participants to generate a more accurate understanding of any given clinical scenario. For this, it is crucial to appreciate what the other party knows or does not know. Psychologists who study the development of language capacities have shown that crucial steps in communication ability come when a child (a) begins to recognize what another person is looking at (a key to understanding the mental model of the other person) and (b) begins to understand what another person cannot perceive or does not know (a key to perceiving dissimilarities between his or her mental model and the other person's) [33]. These effects can be seen even at the neuropsychological level, as in pathbreaking work showing that specific neural circuits in both monkeys and humans are activated when they understand the object of another's action and are quiescent when they do not [34].

These insights into the fundamental psychology of mutual understanding are crucial because they form the basis for perspective-taking [35,36]: understanding a situation, and communicating about it, by taking into account the point of view of the other party, such as a patient being interviewed or a receiving clinician [3,37]. This notion, that taking the perspective of another plays a vital role in the shared meaning of successful communication, has been repeatedly confirmed in vast numbers of studies [36]. Obviously, understanding the perspective of the other party is not a simple procedure and involves great effort from the party that is handing off. If the parties do not appreciate what each individual knows or does not know, the conversation is prone to a host of breakdowns, such as the 'false consensus effect' $[3,38]$, in which a speaker mistakenly believes a listener shares his or her view of the situation, or various forms of misunderstanding that plague teleconferencing and distributed work [39]. A false consensus effect is not unusual in critical care. In our example above, everyone agrees on starting antibiotics and imaging the patient in the event of clinical deterioration, but a false consensus on what 'worse' means may impede this course of actions [40]. If the receiving party does not understand the restarting of pressors as a sign that there may be an alternative explanation for the patient's hypotension, such as sepsis, the receiver may think 'he is getting better, just on low-dose pressors...' and delay diagnostic and therapeutic decisions. Barriers to understanding what the other party knows or does not know may stem from various reasons such as different backgrounds in training (surgeons, internists, and anesthetists working in the same ICU), differences in training level (handoff from an experienced ICU staff to a trainee), or less obvious causes, such as a lack of trust among the participants. 
These multiple lines of social evidence differ in many details, of course, but agree in supporting the value of an enlarged view of handoff, one that highlights not only transmission but also the active co-construction of an understanding of the patient. That understanding can guide subsequent action by the oncoming party and may even change the views and later actions of the party handing off. This kind of high-quality communication about patients can improve patient outcomes [41].

\section{Implications}

There are several implications of this deepened view of handoff communications not as telegrams but as active co-construction of understanding by parties with potentially dissimilar mental models. On this view, it seems unlikely that simple mnemonic tools, such as those that have been increasingly popular in the literature and as quality improvement interventions, will suffice [5]. Little empirical evidence has shown that these tools will, in fact, lead to better continuity of care, efficiency, and safety. Take for example the mnemonic that has been discussed most in the literature, the SBAR tool. SBAR stands for situation, background, assessment, and recommendation. While it may help in providing clarity and a common framework of what should be included in communications, it has no role in helping the parties understand differences in their mental models. In spite of numerous publications, SBAR has failed to show benefits in clinically relevant outcomes. Even simple tasks, such as anticoagulation management [42], did not improve with SBAR, and only modest and clinically indecisive benefits have been demonstrated in clarity of communication during simulation exercises [43] and in order entry errors by surgical residents [44]. More striking is the use of another mnemonic, DeMIST (which stands for Details, Mechanism, Injuries, Signs/Symptoms and Observations, and Treatment given). In one study of this handoff tool [45], the accuracy of emergency department staff recall actually decreased! This empirical evidence actually agrees with qualitative research that suggests that residents perceive standardized tools for handoffs as being possibly detrimental [46]. Owing to the sophisticated nature of handoff encounters, further research on how to incorporate training in handoffs in the medical curriculum and in the health-care culture will be necessary. Several strategies will be important to facilitate the co-construction of a patient trajectory:

1. Encourage and teach the outgoing party to take the perspective of the one coming on [47]. This is especially important in cases in which uncertainty about new information or the expected course of disease is still an issue. In situations in which the nurse or physician handing off has a monopoly of knowledge about the patient, it is easy for a handoff to become a tidy narrative of what was done and why it was justified. Such a narrative can be a seductive goal in a field such as intensive care, in which the uncertainty and the stakes can be extremely high. But this may not be what the oncoming party really needs. The likely problems to be encountered and the unresolved puzzles of the case may be more important for the person taking responsibility. A self-consistent narrative may run the risk of glossing over untidy elements, such as test results that are not completely in line with the most plausible diagnosis. What the new physician may have to decide may not be fully foreshadowed by an account of what has been done and why.

2. Understand the function of questions. One effect of questions is to induce the handing off party, in order to answer intelligibly, to actively take the perspective of the receiver and provide the appropriate information. But questions also often stem from a felt inconsistency as the receiver's image of the patient's situation takes shape. 'What was that sat level?' can be uttered because there was a noise in the room that obscured the spoken number. But it can also occur because the physician has the feeling that the patient he or she is hearing about would normally have been intubated, but that procedure has not been reported, a contradiction to the current mental model that the person possesses. Without this question to the outgoing party, there may be an incomplete understanding about the patient. Sometimes, a question can even be an active formulation of an alternative hypothesis about the patient's fundamental or likely trajectory.

3. Create specific moments to help the incoming party prepare for the handoff, thus shaping his or her mental models of the patients. This may be important, because one of the barriers for an incoming party to exposing inconsistencies in his or her mental model is that it may be insufficiently formed for a discussion during handoffs [4]. Current ways to help with preparations by receiving parties include the pre-transition use of status boards in emergency departments [48] or the developing practice of chart biopsy [49] which is occurring among physicians as they exploit the affordances of new electronic records to begin constructing their own understandings of patients before receiving a handoff.

Overall, the body of research on the active coconstruction of meaning in conversations directs us to examine, and perhaps to expand, the vital role of the receiving party in achieving the full communication required for the quality and safety of patient care.

This article is part of a series on Healthcare Delivery, edited by Dr Andre Amaral and Dr Gordon Rubenfeld. 


\section{Abbreviations}

DeMIST, Details, Mechanism, Injuries, Signs/Symptoms and Observations, and Treatment given; ICU, intensive care unit; SBAR, situation, background, assessment, and recommendation.

\section{Competing interests}

The authors declare that they have no competing interests.

\section{Authors' information}

At the University of Michigan, BH completed his doctoral dissertation on handoffs between emergency and inpatient physicians at the university and has conducted extensive observational research on handoffs [49]. He and MDC have reviewed the entire handoff research literature. ACK-BA is a clinician in quality improvement at Sunnybrook Health Sciences Centre, Department of Critical Care Medicine, and is currently conducting several studies on handoffs in critical care.

\section{Acknowledgments}

The authors are grateful for the financial support of the Robert Wood Johnson Foundation (Investigator Award R36HS018758), ProQuest (Ann Arbor, MI, USA), and the Agency for Healthcare Research and Quality (grant R36HS018758). The content is solely the responsibility of the authors and does not necessarily represent the official views of the Agency for Healthcare Research and Quality.

\section{Author details}

'School of Information, University of Michigan, 3442 North Quad, 105 South State Street, Ann Arbor, Ml 48109-1285, USA. '2Division of Health Services Management and Policy, College of Public Health, Ohio State University, $200 \mathrm{C}$ Cunz Hall, 1841 Neil Avenue, Columbus, OH 43210-1351, USA. ${ }^{3}$ Department of Critical Care Medicine, Sunnybrook Health Sciences Centre, University of Toronto, 2075 Bayview Avenue, Room D108, Toronto, ON M4N 3M5, Canada.

\section{Published: 8 February 2012}

\section{References}

1. Cohen MD, Hilligoss PB: The published literature on handoffs in hospitals: deficiencies identified in an extensive review. Qual Saf Health Care 2010, 19:493-497.

2. Farnan J, Paro J, Rodriguez R, Reddy S, Horwitz L, Johnson J, Arora V: Hand-off education and evaluation: piloting the observed simulated hand-off experience (OSHE). J Gen Intern Med 2010, 25:129-134.

3. Chang VY, Arora VM, Lev-Ari S, D'Arcy M, Keysar B: Interns overestimate the effectiveness of their hand-off communication. Pediatrics 2010, 125:491-496.

4. Patterson ES, Wears RL: Patient handoffs: standardized and reliable measurement tools remain elusive. Jt Comm J Qual Patient Saf 2010, 36:52-61.

5. Riesenberg LA, Leitzsch J, Little BW: Systematic review of handoff mnemonics literature. Am J Med Qual 2009, 24:196-204

6. Petersen LA, Brennan TA, O'Neil AC, Cook EF, Lee TH: Does housestaff discontinuity of care increase the risk for preventable adverse events? Ann Intern Med 1994, 121:866-872.

7. Gawande AA, Zinner MJ, Studdert DM, Brennan TA: Analysis of errors reported by surgeons at three teaching hospitals. Surgery 2003, 133:614-621.

8. Gandhi TK, Kachalia A, Thomas EJ, Puopolo AL, Yoon C, Brennan TA, Studdert DM: Missed and delayed diagnoses in the ambulatory setting: a study of closed malpractice claims. Ann Intern Med 2006, 145:488-496.

9. Kachalia A, Gandhi TK, Puopolo AL, Yoon C, Thomas EJ, Griffey R, Brennan TA, Studdert DM: Missed and delayed diagnoses in the emergency department: a study of closed malpractice claims from 4 liability insurers. Ann Emerg Med 2007, 49:196-205.

10. The Joint Commission: National Patient Safety Goals: 2006 Critical Access Hospital and Hospital National Patient Safety Goals. Oakbrook Terrace, IL: The Joint Commission; 2006

11. Eisenberg EM, Murphy AG, Sutcliffe K, Wears R, Schenkel S, Perry S, Vanderhoef M: Communication in emergency medicine: implications for patient safety. Communication Monographs 2005, 72:390-413.

12. Engesmo J, Tjora AH: Documenting for whom? A symbolic interactionist analysis of technologically induced changes of nursing handovers. New Technology, Work and Employment 2006, 21:176-189.
13. Patterson ES, Wears RL: Beyond'communication failure.'Ann Emerg Med 2009, 53:711-712.

14. Chu ES, Reid M, SchulzT, Burden M, Mancini D, Ambardekar AV, Keniston A, Albert RK: A structured handoff program for interns. Acad Med 2009, 84:347-352.

15. Committee on Patient Safety and Quality Improvement: ACOG committee opinion. Number 367. June 2007. Communication strategies for patient handoffs. Obstet Gynecol 2007, 109:1503-1505.

16. Greenberg CC, Regenbogen SE, Studdert DM, Lipsitz SR, Rogers SO, Zinner MJ, Gawande AA: Patterns of communication breakdowns resulting in injury to surgical patients. J Am Coll Surg 2007, 204:533-540.

17. The Joint Commision, Joint Commision International, World Health Organization: Communication During Patient Hand-Overs. Geneva: World Health Organization; 2007.

18. Horwitz LI, Moin T, Krumholz HM, Wang L, Bradley EH: What are covering doctors told about their patients? Analysis of sign-out among internal medicine house staff. Qual Saf Health Care 2009, 18:248-255.

19. Shannon CE: The Mathematical Theory of Communication. Urbana, IL: University of Illinois Press; 1949.

20. Brannen ML, Cameron KA, Adler M, Goodman D, Holl JL: Admission handoff communications: clinician's shared understanding of patient severity of illness and problems. Journal of Patient Safety 2009, 5:237-242.

21. Hsu Y-chen: The effects of metaphors on novice and expert learners' performance and mental-model development. Interacting with Computers 2006, 18:770-792.

22. Berlo DK: The Process of Communication: An Introduction to Theory and Practice. New York: Holt, Rinehart and Winston; 1960

23. PlaxTG, Rosenfeld LB: Receiver differences and the comprehension of spoken messages. Journal of Experimental Education 1979, 48:23-28,

24. Clark HH, Wilkes-Gibbs D: Referring as a collaborative process. Cognition 1986, 22:1-39.

25. Harris T: Applied Organizational Communication: Theory and Practice in a Global Environment. 3rd edition. New York: L. Erlbaum Associates; 2008

26. Littlejohn SW: Theories of Human Communication. Belmont, CA: Wadsworth Pub. Co: 1992

27. Clark HH: Using Language. New York: Cambridge University Press; 1996.

28. Johnson-Laird PN: The perception and memory of sentences. In New Horizons in Linquistics. Edited by Lyons J. Hillsdale, NJ: Penguin; 1970.

29. Mohammed S, Ferzandi L, Hamilton K: Metaphor no more: a 15-year review of the team mental model construct. Journal of Management 2010, 36:876 -910 .

30. DeChurch $L A$, Mesmer-Magnus JR: The cognitive underpinnings of effective teamwork: a meta-analysis. Journal of Applied Psychology 2010, 95:32-53.

31. Westli H, Johnsen B, Eid J, Rasten I, Brattebø G: Teamwork skills, shared mental models, and performance in simulated trauma teams: an independent group design. Scand J Trauma Resusc Emerg Med 2010, 18:1-8.

32. Mishra A, Catchpole K, Dale T, McCulloch P: The influence of non-technical performance on technical outcome in laparoscopic cholecystectomy. Surg Endosc 2008, 22:68-73.

33. Tomasello M: Constructing a Language: A Usage-Based Theory of Language Acquisition. Cambridge, MA: Harvard University Press; 2003.

34. Rizzolatti G, Sinigaglia C: Mirrors in the Brain: How Our Minds Share Actions and Emotions. Oxford: Oxford University Press; 2008

35. Krauss RM, Fussell SR: Perspective-taking in communication: representations of others' knowledge in reference. Social Cognition 1991 9:2-24.

36. Mason MF, Macrae CN: Perspective-taking from a social neuroscience standpoint. Group Processes \& Intergroup Relations 2008, 11:215-232.

37. Blatt B, LeLacheur SF, Galinsky AD, Simmens SJ, Greenberg L: Does perspective-taking increase patient satisfaction in medical encounters? Acad Med 2010, 85:1445-1452.

38. Marks $\mathrm{G}$, Miller N: Ten years of research on the false-consensus effect: an empirical and theoretical review. Psychological Bulletin 1987, 102:72-90.

39. Bruni A, Gherardi S, Parolin LL: Knowing in a system of fragmented knowledge. Mind, Culture, and Activity 2007, 14:83-102.

40. Mukherjee S: A precarious exchange. N Engl J Med 2004, 351:1822-1824.

41. Vogus TJ, Sutcliffe KM: The safety organizing scale: development and validation of a behavioral measure of safety culture in hospital nursing units. Med Care 2007, 45:46-54.

42. Field TS, Tjia J, Mazor KM, Donovan JL, Kanaan AO, Harrold LR, Reed G, Doherty P, Spenard A, Gurwitz JH: Randomized trial of a warfarin 
communication protocol for nursing homes: an SBAR-based approach Am J Med 2011, 124:179.e1-179.e7.

43. Marshall S, Harrison J, Flanagan B: The teaching of a structured tool improves the clarity and content of interprofessional clinical communication. Qual Saf Health Care 2009, 18:137-140.

44. Telem DA, Buch KE, Ellis S, Coakley B, Divino CM: Integration of a formalized handoff system into the surgical curriculum: resident perspectives and early results. Arch Surg 2011, 146:89-93.

45. Talbot $R$, Bleetman A: Retention of information by emergency department staff at ambulance handover: do standardised approaches work? Emerg Med J 2007, 24:539-542.

46. Philibert I: Use of strategies from high-reliability organisations to the patient hand-off by resident physicians: practical implications. Qual Saf Health Care 2009, 18:261-266.

47. Epley N, Caruso EM: Perspective taking: misstepping into others' shoes.
In Handbook of Imagination and Simulation. Edited by Markman KD, Klein WM, Suhr JA. New York: Psychology Press; 2009:313-330.

48. Wears RL, Perry SJ, Wilson S, Galliers J, Fone J: Emergency department status boards: user-evolved artefacts for inter- and intra-group coordination. Cogn Tech Work 2006, 9:163-170

49. Hilligoss B: Dissecting the pre-handoff chart biopsy: information seeking in the electronic health record. Paper presented at: American Society for Information Science and Technology 2010 Annual Meeting; 22-27 Oct. 2010; Pittsburgh, PA.

doi:10.1186/cc10536

Cite this article as: Cohen MD, et al:: A handoff is not a telegram: an understanding of the patient is co-constructed. Critical Care 2012, 16:303. 